\title{
Synthesis of the neural coordinated control algorithm for the model of CNC machine
}

\author{
Valeriy Lyubich ${ }^{1, *}$, Vladimir Frolov ${ }^{1}$ and Vladimir Beliaev ${ }^{2}$ \\ ${ }^{1}$ Peter the Great St. Petersburg Ploytechnic University, Saint-Petersburg, Russia \\ ${ }^{2}$ North-West Open University, Yakornaya 9a, 195027 Saint-Petersburg, Russia
}

\begin{abstract}
Objectives: Increase quality factor of the CNC machine model in comparison with the Uncoupled System by synthesizing Neural Coordinated Control. Synthesis: We synthesized the Neural Coordinated Control algorithm based on the coordinated control algorithm and neural control. Experiment: Using mathematical modeling we compared the synthesized algo-rithms and the uncoupled system using the following criteria: contour error, contour speed error, and score function. Results: The four NCC algorithms were synthesized and trained. The experiment shows that synthesized algorithms have better score function values and better quality factor values in comparison to the reference Uncoupled System. Conclusion: The quality factor of the CNC machine model was successfully in-creased by using the synthesized Neural Coordinated Control algorithm.
\end{abstract}

\section{Introduction}

Increase of quality factor - ratio of contour speed to contour error - of contour tracking is a relevant task, because systems with high quality factor can make more accurate operations or make the same operations faster, that increases performance of the machine [1-6].

Modern developments in computing technologies allow us to implement on practice more computation complex algorithms, for example: neural network control [7-10], coordinated control algorithms [1-3, 11, 12], fuzzy logic control [13,14], and other [15-18].

In this paper we propose the neural coordinated control (NCC) which is based on the coordinated control algorithm and neural control. This combination is chosen by the following reasons:

- The coordinated control algorithm is an algorithm with the coupled structure which has several advantages over systems with the uncoupled structure (for example the Uncouple System) [1,4-6]: contour error is minimized directly - the Uncoupled System minimizes contour error indirectly through minimizations of coordinated errors; it is possible to set control priorities by choosing ratio of contour error and contour speed error. In many cases low contour error is preferable to low contour speed error; etc [4];

- Neural network regulators can be used to form complex non-linear combinations of input parameters on its outputs. This can be used to create unique and specialized control for specific control tasks with relatively small amount of dynamic information [7-9].
The goal of the paper: Increase quality factor of the CNC machine model in comparison with the Uncoupled System by using Neural Coordinated Control.

The tasks:

1.Synthesize several NCC with different neural network structures and different training sets;

2. Train the neural networks using the chosen score function and gradient descent learning algorithm;

Compare the synthesized algorithms and the Uncoupled System using the following criteria: the root mean square quality factor, the minimum quality factor, the root mean square contour speed error and the score function.

\section{Synthesis}

The simplified CNC machine model (1) fig. 1 has been chosen as the plant.

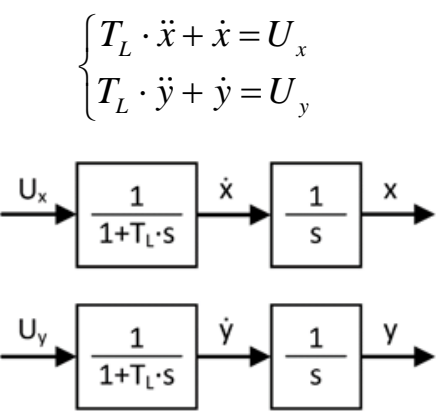

Fig. 1. The plant structure.

As previously been mentioned the NCC is based on the coordinated control algorithm $[1,2,4,19]$. The main

\footnotetext{
* Corresponding author: valeriy.lyubich@gmail.com
} 
idea of the coordinated control algorithm is forming the speed control vector as combination of two vectors fig. 2: the tangent speed $\left(\mathrm{V}_{\tau}\right)$, which sets movement along the trajectory, and the normal speed $\left(V_{n}\right)$, which minimizes contour error.

Contour error $\left(E_{k}\right)$ - minimal distance between the end effector position and the trajectory.

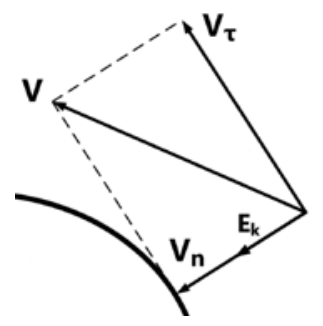

Fig. 2. The speed control vector as a combination of the tangent speed and the normal speed.

The structure of NCC is shown on the Fig. 3.

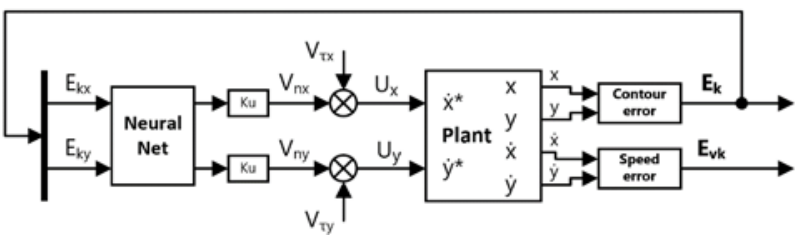

Fig. 3. The structure of NCC.

The trajectory is the circular arc:

$$
y^{2}+x^{2}=R^{2}
$$

The tangent speed for circular arc:

$$
\left\{\begin{array}{l}
V_{\tau x}=V_{k} * \cos \left(\frac{\pi}{2}-\tan ^{-1}\left(\frac{y}{x}\right)\right) \\
V_{\tau y}=-V_{k} * \sin \left(\frac{\pi}{2}-\tan ^{-1}\left(\frac{y}{x}\right)\right)
\end{array}\right.
$$

The neural regulator for NCC has one of these structures fig. 4. They are used to form the normal speed (4), where $K_{u}$ is a positive gain.

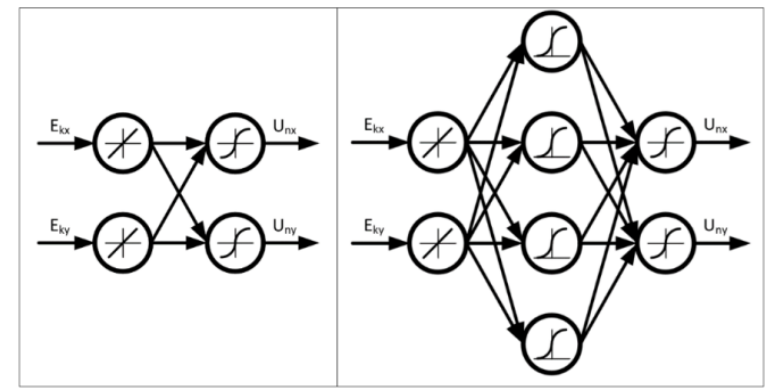

Fig. 4. The structures of the neural regulators. Left - neural network without hidden layers; Right - neural network with one hidden layer.

$$
\left\{\begin{array}{l}
V_{n x}=K_{u} \cdot U_{n x} \\
V_{n y}=K_{u} \cdot U_{n y}
\end{array}\right.
$$

The neural networks are trained using gradient descent learning algorithm [20]. The learning criterion or the score function (5) is the linear convolution [21] of the two criteria: the root mean square of contour error and the root mean square of contour speed error. In practice a low value of contour speed is usually preferable to a low value of contour speed error, hence $\alpha_{2}$ is chosen to be lower than $\alpha_{1}$.

$$
\text { Score }=\alpha_{1}\left\langle E_{k}\right\rangle+\alpha_{2}\left\langle E_{v k}\right\rangle
$$

Contour error $\left(E_{k}\right)$ and contour speed error $\left(E_{v k}\right)$ for the circular arc are:

$$
\left\{\begin{array}{l}
E_{k}=\sqrt{x^{2}+y^{2}}-R \\
E_{v k}=\dot{x}^{2}+\dot{y}^{2}-V_{k}^{2}
\end{array}\right.
$$

\section{Experiment}

The experiment was conducted using mathematical model calculation.

We synthesized and trained four algorithms: NR_L0E1, NR_L0E3, NR_L1E1 and NR_L1E3, where:

- L0 and L1 - number of hidden layers: 0 and 1 accordingly;

- E1 and E3 - size of training set: 1 and 3 elements accordingly.

The synthesized algorithms were compared with the Uncoupled System fig. 5. .

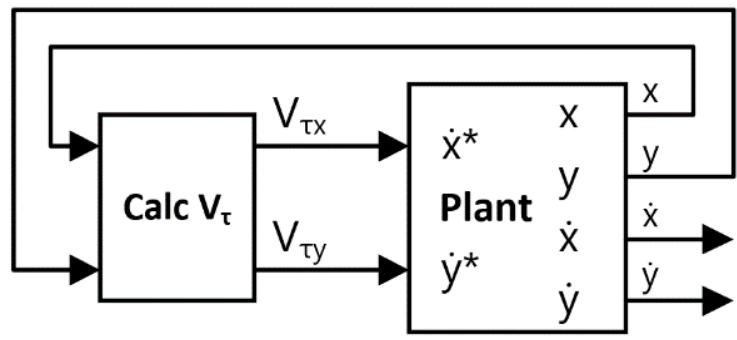

Fig. 5. The structure of the reference Uncoupled System.

Parameters:

- The plant parameter fig. 1 :

$T_{L}=0.001[s]$

- Contour speed for all algorithms $(3,6)$

$V_{k}=0.4[\mathrm{~m} / \mathrm{s}]$

- The training sets (2):

$\mathrm{E} 1: \mathrm{R}=9$ [m]

E3: $\mathrm{R}=9,11,13[\mathrm{~m}]$

- The initial position, speed and residuals:

$x_{0}=-R[\mathrm{~m}] ; \quad y_{0}=0[\mathrm{~m}] ; \quad E_{k 0}=0$

$\dot{x}_{0}=0[\mathrm{~m} / \mathrm{s}] ; \quad \dot{y}_{0}=0[\mathrm{~m} / \mathrm{s}] ; \quad E_{v k 0}=-V_{k}^{2}$

- The neural regulator gain (4):

$K_{u}=0.8$

- The score coefficients (5):

$\alpha_{1}=1 ; \quad \alpha_{2}=0.001$

- The simulation time:

$T_{\text {sim }}=10[\mathrm{~s}]$ 
- Initiation of the neural networks weights and biases:

Weights: $\mathrm{W}_{\mathrm{ij}}=-0.1 \ldots 0.1$

Biases: $b_{i j}=-0.1 \ldots 0.1$

Since the score function is generally not convex [10] - i.e. have several local minimums - the neural networks were initialized randomly and were trained several times.

The experiment results are presented in table 1.

Table 1. The results of the experiment.

\begin{tabular}{|c|c|c|c|c|c|}
\hline \multirow{4}{*}{ Type } & $\begin{array}{c}\text { R, } \\
\mathrm{m}\end{array}$ & Score & $<v>, 1 / \mathrm{s}$ & $\begin{array}{c}<\text { Evk }>, \\
\mathrm{m} / \mathrm{s}\end{array}$ & $\begin{array}{c}\text { v_min, } \\
1 / \mathrm{s}\end{array}$ \\
\hline \multirow{5}{*}{ US } & 9 & 0.0287 & 13.9373 & 0.0080 & 7.8125 \\
\cline { 2 - 6 } & 10 & 0.0259 & 15.4440 & 0.0080 & 8.6768 \\
\cline { 2 - 6 } & 11 & 0.0235 & 17.0213 & 0.0080 & 9.5465 \\
\cline { 2 - 6 } & 12 & 0.0216 & 18.5185 & 0.0080 & 10.3896 \\
\cline { 2 - 6 } NR_L0E1 & 13 & 0.0199 & 20.1005 & 0.0080 & 11.2676 \\
\hline \multirow{5}{*}{ NR_L0E3 } & 9 & 0.0086 & 46.5116 & 0.0242 & 17.8571 \\
\cline { 2 - 6 } & 10 & 0.0050 & 80.0000 & 0.0241 & 29.1971 \\
\cline { 2 - 6 } & 11 & 0.0034 & 117.6471 & 0.0241 & 59.7015 \\
\cline { 2 - 6 } & 12 & 0.0044 & 90.9091 & 0.0241 & 66.6667 \\
\cline { 2 - 6 } & 13 & 0.0062 & 64.5161 & 0.0241 & 50.6329 \\
\cline { 2 - 6 } & 9 & 0.0019 & 222.2222 & 0.0257 & 78.4314 \\
\cline { 2 - 6 } & 11 & 0.0024 & 173.9130 & 0.0257 & 50.0000 \\
\cline { 2 - 6 } & 12 & 0.0044 & 117.6471 & 0.0258 & 38.4615 \\
\hline \multirow{5}{*}{ NR_L1E1 } & 9.0054 & 74.0749 & 0.0259 & 32.2581 \\
\cline { 2 - 6 } & 10 & 0.0082 & 48.7805 & 0.0259 & 28.5714 \\
\cline { 2 - 6 } & 11 & 0.0047 & 85.1064 & 0.0120 & 19.7044 \\
\cline { 2 - 6 } & 12 & 0.0038 & 133.3333 & 0.0120 & 61.7460 \\
\cline { 2 - 6 } & 13 & 0.0055 & 72.7273 & 0.0119 & 76.9231 \\
\hline \multirow{5}{*}{ NR_L1E3 } & 9 & 0.0131 & 30.5344 & 0.0119 & 57.9710 \\
\cline { 2 - 6 } & 10 & 0.0095 & 42.1053 & 0.0105 & 14.1343 \\
\cline { 2 - 6 } & 11 & 0.0065 & 61.5385 & 0.0105 & 25.9749 \\
\cline { 2 - 6 } & 12 & 0.0042 & 95.2381 & 0.0105 & 37.7358 \\
\cline { 2 - 6 } & 13 & 0.0025 & 160.0000 & 0.0105 & 60.6061 \\
\hline
\end{tabular}

Using data from table 1 we plotted comparative graphs fig. 6.

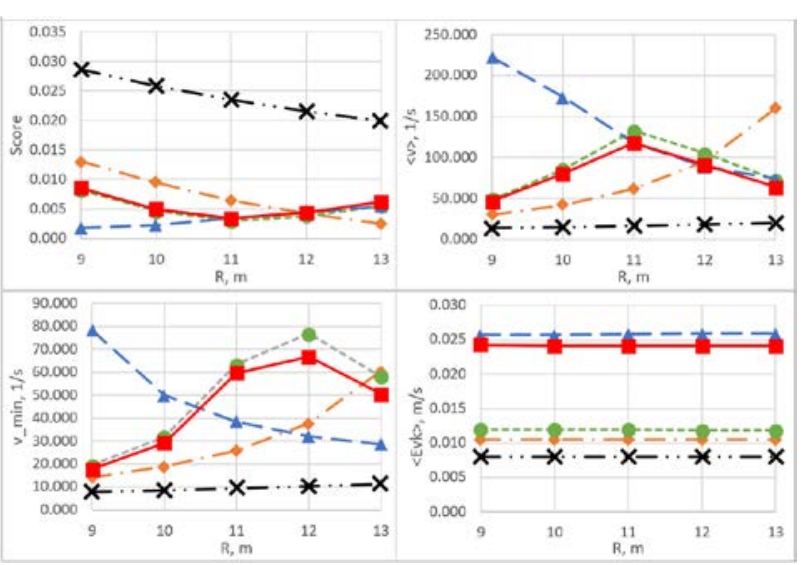

Fig. 6. The experimental comparative graphs. Top Left - The score function to radius (of the circular arc); Top Right - The root mean square quality factor to radius; Bottom Left - The minimal quality factor to radius; Bottom Right - The root mean square contour speed error to radius. Legend: US - the black dash-dot-dotted line (-..-) with cross markers (x) ; NR_L0E1 the red solid line (-) with square markers $(\square)$; NR_LOE3 the blue dashed line (--) with triangular markers $(\Delta)$;

NR_L1E1 - the green dotted line with circular markers (O) ; NR_L1E3 - the orange dash-dotted line with diamond markers $(\diamond)$

\section{Results}

The four NCC algorithms were synthesized and trained. The experiment table 1 and fig. 6 shows that synthesized algorithms have better score function values and better quality factor values in comparison to the reference Uncoupled System. The synthesized algorithms have lower contour speed error than the Uncoupled System it is a consequence of choosing the relatively small score function (3) coefficient $\alpha_{2}=0.001$.

None of the synthesized algorithms is pareto optimal by the score function. It is probably connected with the non-convexity of the score function, i.e. during learning process the neural regulators got into local minimums

\section{Conclusion}

From the results we can conclude that the quality factor of the CNC machine model was increased - in comparison to the Uncoupled System - by using the Neural Coordinated Control.

Choosing more complex neural network or having more training examples does not lead to a pareto optimal by score solution. The reason behind this may be nonconvexity of the score function.

The synthesized algorithms have worse - than the Uncoupled System - speed error due to the chosen score coefficients.

\section{Discussion}

The score function has coefficients which might be used to set control priorities, i.e. how does these coefficients influent the criteria?

How more complex neural network's structures and bigger training sets will influence the criteria?

The neural networks might be trained using different algorithms, for example using a generic algorithm, which should better handle non-convex score functions

\section{References}

1. R. Lawrence and C. Heron, in 2016 IEEE Pulp, Pap. For. Ind. Conf. (IEEE, 2016), pp. 174-181

2. S. Rachev, L. Dimitrov, K. Karakoulidis, I. D. Ivanov, and C.-V. Anghel Drugarin, in 2018 Int. Conf. Appl. Theor. Electr. (IEEE, 2018), pp. 1-6

3. E. Ikonen and P. Heikkinen, Neural Comput. Appl. 9, 165 (2000)

4. N. P. Ovchinnikov, Journal of mining institute. 235, 65 (2019)

5. P. M. Widodo and D. Rinaldy, J. Eng. Sci. Technol. 14, 1055 (2019)

6. A. Chusov, G. Podporkin, M. Pinchuk, D. Ivanov, I. Murashov, and V. Frolov, in 2016 33rd Int. Conf. Light. Prot. (IEEE, 2016), pp. 1-9

7. I. Murashov, V. Frolov, and D. Ivanov, in 2016 IEEE NW Russ. Young Res. Electr. Electron. Eng. Conf. (IEEE, 2016), pp. 625-628 
8. O. B. Shonin and V. S. Pronko, Journal of mining institute. 218, 270 (2016)

9. N. V. Obraztsov, D. I. Subbotin, V. E. Popov, V. Y. Frolov, and A. V. Surov, J. Phys. Conf. Ser. 1038, 012137 (2018)

10. I. S. Churkin, D. Ivanov, V. Frolov, and D. Uhrlandt, in 19th Symp. Phys. Switch. Arc 2011, FSO 2011 (2011)

11. V. I. Aleksandrov and Jerzy Sobota, Journal of mining institute. 213, 9 (2015)

12. R. Tao, R. Xiao, and W. Liu, Proc. Inst. Mech. Eng. Part A J. Power Energy (2018)

13. H. Sun, S. Yuan, Y. Luo, and Y. Guo, Paiguan Jixie Gongcheng Xuebao/Journal Drain. Irrig. Mach. Eng. (2016)

14. Y. D. Khechuev, B. E. Kalashnikov, and V. I. Ol\&apos;shevskii, Russ. Electr. Eng. (2006)

15. M. Zagirnyak, Przegląd Elektrotechniczny 1, 106 (2019)

16. K. A. Tahboub, M. I. Albakri, and A. M. Arafeh, in Vol. 4 ASME/IEEE Int. Conf. Mechatron. Embed. Syst. Appl. 19th Reliab. Stress Anal. Fail. Prev. Conf. (ASME, 2007), pp. 209-217

17. L. H. de Paula, F. C. Storti, and E. Fortaleza, IFACPapersOnLine 48, 33 (2015)

18. A. Makarov and M. Kukhtik, in 2018 Int. Ural Conf. Green Energy (IEEE, 2018), pp. 265-269

19. Z. B. Jiang, T. Zhong, and Y. H. Rao, in 2011 Int. Conf. Inf. Technol. Comput. Eng. Manag. Sci. (IEEE, 2011), pp. 131-135

20. Artyukhov, I. I. Bochkareva, and S. V. Molot, in 2014 Int. Conf. Actual Probl. Electron Devices Eng. (IEEE, 2014), pp. 11-17 